J. Phys. IV France 127 (2005) 157-162

(C) EDP Sciences, Les Ulis

DOI: $10.1051 /$ jp4:2005127024

\title{
Caractérisation du seuil d'ablation des parois dans les sources de rayonnement EUV par décharge capillaire
}

\author{
O. Sarroukh ${ }^{1}$, E. Robert ${ }^{1}$, J. Kaiser ${ }^{2}$, R. Viladrosa ${ }^{1}$, J.M. Pouvesle ${ }^{1}$, \\ C. Fleurier ${ }^{1}$ et C. Cachoncinlle ${ }^{1}$ \\ ${ }^{1}$ GREMI (Groupe de Recherche sur l'Énergétique des Milieux lonisés) : Polytech Orléans, \\ Université d'Orléans, 14 rue 'Issoudun, BP. 6744, 45067 Orléans Cedex 2, France \\ 2 Institute of Physical Engineering, Brno University of Technology, Technická 2, 61669 Brno, \\ République Tchéque
}

\begin{abstract}
Résumé. Les sources de rayonnement Extrême Ultraviolet (EUV) par décharge capillaire connaissent un intérêt de plus en plus considérable pour de nombreuses applications scientifiques et technologiques. Au GREMI, nous étudions le fonctionnement des sources de rayonnement EUV émettant à $13.5 \mathrm{~nm}$ pouvant répondre à certaines applications liées à la nouvelle génération de la lithographie. Ces sources pulsées, nommées CAPELLA, ATגAS et PROXIMA, sont basées sur une décharge capillaire en flux de gaz. Ce type de décharge produit un plasma chaud, dense et fortement ionisé émettant dans la gamme spectrale de l'EUV. En effet, les températures électroniques peuvent atteindre des dizaines d'électronvolts et les densités électroniques sont de l'ordre de $10^{17} \mathrm{~cm}^{-3}$. Les échanges thermiques entre le plasma ainsi créé et les parois du capillaire peuvent être suffisamment important pour que les parois du capillaire soient ablatées. Cette ablation peut être plus ou moins importante selon la densité d'énergie injectée dans le capillaire. Des mesures spectroscopiques du plasma émetteur dans les gammes spectrales de l'UV et l'EUV ont montré la présence d'un seuil d'ablation situé autour de à $350 \mathrm{~J} . \mathrm{cm}^{-3}$. Nous utilisons un modèle thermique présenté [1] pour modéliser l'évolution temporelle de la température des parois du capillaire. Le seuil peut être ainsi estimé. Les résultats expérimentaux obtenus à partir de nos mesures spectroscopiques sont comparés ensuite aux résultats numériques.
\end{abstract}

\section{INTRODUCTION}

Actuellement, de nombreux travaux de recherche sont menés en France et dans le monde entier pour développer des sources de rayonnement émettant à des longueurs d'onde de plus en plus faibles capables de répondre à diverses applications scientifiques et technologiques.

Au GREMI, nous étudions le fonctionnement des sources de rayonnement EUV émettant à $13.5 \mathrm{~nm}$ pouvant satisfaire certaines applications liées à la nouvelle génération de la lithographie EUV [2]. Plusieurs sources pulsées nommées CAPELLA, AT AAs et PROXIMA ont été développées. Ces sources sont basées sur un même principe: le plasma émetteur est généré par décharge dans un capillaire en alumine $\mathrm{Al}_{2} \mathrm{O}_{3}$ où circule un gaz (xénon $\mathrm{Xe}$, hélium He, propène $\mathrm{C}_{3} \mathrm{H}_{6} \ldots$ ) à basse pression. Les plasmas générés dans ce type de décharge sont très denses et chauds; la température électronique du plasma peut atteindre des dizaines d'électronvolts et la densité électronique est de l'ordre de $10^{17} \mathrm{~cm}^{-3}$.

Le plasma émetteur est étudié par spectroscopie à la fois intégrée et résolue en temps. Ces mesures permettent d'identifier les différents ions présents dans le milieu et notamment de suivre leur évolution temporelle. Soulignons qu'il est important de maîtriser et de minimiser l'ablation des parois du capillaire pour assurer une longue durée de vie des sources EUV basées sur ce concept. Pour ce faire, nous nous sommes particulièrement intéressés à l'étude de l'évolution des espèces issus de l'ablation du capillaire en fonction de certains paramètres de la décharge. L'évolution de l'intensité de certaines raies d'aluminium issu de l'alumine qui constitue les parois sera étudiée en fonction de la densité d'énergie injectée dans le capillaire. Ainsi, nous montrons que cette évolution manifeste la présence d'un seuil d'ablation, situé

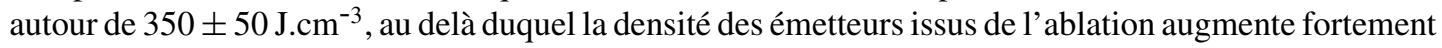


et par conséquent le processus d'ablation doit être pris en considération dans l'évolution du plasma. Nous montrons ensuite l'influence du gaz circulant dans le capillaire sur ce seuil d'ablation des parois.

Le travail présenté en [1] décrit un modèle thermique capable d'estimer l'évolution de la température des parois du capillaire lors de la décharge. Il prédit que le seuil d'ablation des parois du capillaire est atteint quand la température des parois devient égale à sa température de fusion. Nous montrons que ce seuil dépend principalement des propriétés thermiques du capillaire et du temps pendant lequel l'énergie est transférée au plasma. Les résultats numériques issus du modèle seront comparés aux résultats expérimentaux.

\section{DISPOSITIF EXPÉRIMENTAL}

Le dispositif expérimental de la source de rayonnement EUV a été présenté antérieurement en détail [2]. Le dispositif expérimental regroupe principalement quatre éléments: l'alimentation haute tension, le banc de condensateurs de 2 à $24 \mathrm{nF}$ chargé à des tensions pouvant atteindre $25 \mathrm{kV}$, le thyratron pour déclencher la décharge et le capillaire en oxyde d'aluminium, alumine $\mathrm{Al}_{2} \mathrm{O}_{3}$, d'un diamètre de $1 \mathrm{~mm}$ et $10 \mathrm{~mm}$ de longueur. L'énergie électrique, stockée dans les bancs de condensateurs, peut atteindre $6 \mathrm{~J}$,

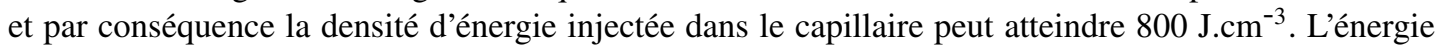
électrique est dissipée dans le circuit de commutation et dans le capillaire. Dans le cas de notre dispositif, le plasma créé dans le capillaire constitue un milieu fortement résistif par rapport à celui créé dans le commutateur. Ainsi, l'énergie stockée est donc principalement dissipée dans le capillaire.

Le rayonnement émis est analysé au moyen de deux spectromètres, l'un couvrant le domaine spectral de l'EUV et l'autre allant de l'UV jusqu'à l'infrarouge. Le spectromètre EUV est muni dans son plan focale d'un détecteur MCP pouvant opérer en mode continu ou mode pulsé. Il convertit le rayonnement EUV en rayonnement Visible. Une caméra CCD intensifiée permet ensuite d'enregistrer les spectres d'émission résolus ou intégrés en temps du rayonnement émis par le plasma.

\section{SPECTROSCOPIE EUV ET UV DU PLASMA}

L'émission du plasma créé par décharge est étudiée par spectroscopie résolue ou intégrée en temps dans les domaines spectraux de l'EUV entre 18 à 32m et de l'ultraviolet. Le plasma créé dans le capillaire est constitué essentiellement du gaz injecté dans le capillaire, de l'air résiduel et des éléments issus de l'ablation des parois. Soulignons que l'oxygène présent dans le spectre du rayonnement émis par la décharge peut être issu à la fois de l'air résiduel et de l'alumine qui constitue le capillaire. Par conséquent, seule, la présence d'aluminium dans le spectre représente la signature de l'ablation. Le gaz utilisé dans les expériences rapportées dans ce paragraphe est l'hélium. Il est injecté continuellement dans le capillaire et sa pression est maintenue à 2 mbar. L'hélium présente une faible émissivité par rapport à d'autres gaz tel que le xénon qui est le plus souvent employé dans les sources dédiées à la nouvelle génération de la lithographie EUV. L'utilisation d'hélium (pauvre en raie d'émission) facilite ainsi l'identification des raies d'émission des élément issus de l'ablation des parois dans le spectre.

\subsection{Spectroscopie UV intégrée en temps}

Nous présentons en figure 1 deux spectres intégrés en temps du rayonnement émis entre 382 et $405 \mathrm{~nm}$ correspondant à deux densités d'énergie de 300 et $500 \mathrm{~J} . \mathrm{cm}^{-3}$. Les spectres ont été réalisés dans le même conditions, seule l'énergie stockée a été modifiée.

Nous constatons que les deux spectres sont complètement différents. Le spectre correspondant à $300 \mathrm{~J} . \mathrm{cm}^{-3}$ révèle la présence de plusieurs raies d'oxygène issus essentiellement de l' aire résiduel présent dans le capillaire. Le spectre correspondant à $500 \mathrm{~J} . \mathrm{cm}^{-3}$ manifeste une domination très marquée des raies de résonance d'aluminium neutre Al I à 394.4 et $396.1 \mathrm{~nm}$. L'ablation de parois du capillaire ne peut être négligée dans ce second cas. 


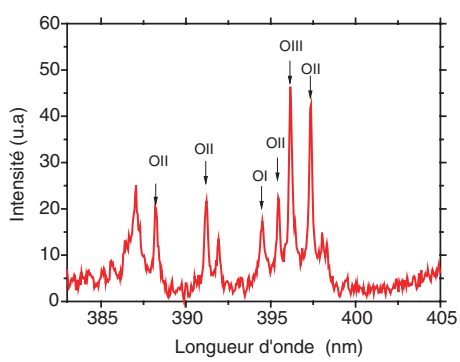

a)

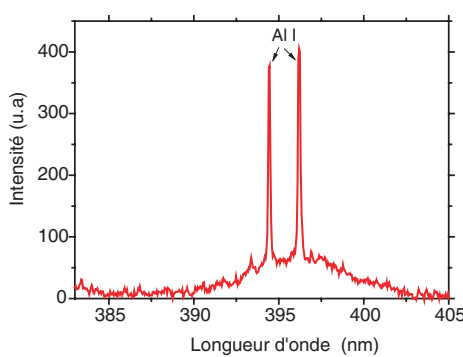

b)

Figure 1. Spectres intégrés en temps du rayonnement UV émis par la décharge pour deux densité d'énergie injecté dans le capillaire correspondant à $300 \mathrm{~J} . \mathrm{cm}^{-3}$ (a) et à $500 \mathrm{~J} . \mathrm{cm}^{-3}$ (b).

A noter que l'émission du plasma dans cette plage de longueur d'onde est dix fois plus importante dans le deuxième cas ce qui est dû en partie à l'augmentation de la densité d'émetteurs issue de l'ablation des parois.

\subsection{Spectroscopie EUV intégrée en temps}

On présente en figure 2 un spectre de rayonnement émis par la décharge dans la gamme de 18 à $32 \mathrm{~nm}$ correspondant à une densité d'énergie de $600 \mathrm{~J} . \mathrm{cm}^{-3}$. Nous remarquons que le spectre est complètement dominé par plusieurs raies d'émission correspondant à plusieurs états d'ionisation d'aluminium (jusqu'à six fois ionisé).

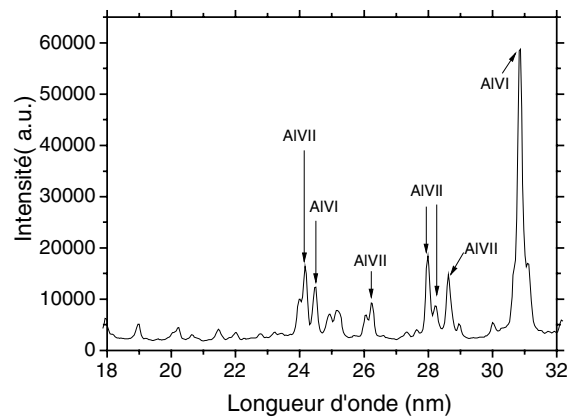

Figure 2. Spectre intégré en temps du rayonnement EUV émis dans la gamme de 18 à $32 \mathrm{~nm}$ pour une densité d'énergie injecté de $600 \mathrm{~J} . \mathrm{cm}^{-3}$.

Les spectres résolus en temps, réalisés dans ces mêmes conditions, ont montré une domination des raies d'aluminium dans cette plage spectrale dès 100 ns après l'établissement du courant de la décharge. Par exemple, l'intensité de la raie d'aluminium à $30.8 \mathrm{~nm}$ est maximale à $120 \mathrm{~ns}$. Notons que la raie d'hélium He II à $30.3 \mathrm{~nm}$ a été identifiée uniquement dans les spectres résolus en temps enregistrés au début de décharge (40 ns). Ces mesures corroborent les résultats obtenus dans l'UV.

\subsection{Observation du seuil d'ablation des parois du capillaire}

Nous étudions dans ce paragraphe l'influence de l'énergie injectée dans le capillaire sur l'intensité des raies d'aluminium Al I à 396.1nm et Al VI à 30.8 nm que nous avons identifiées sur les spectres présentés précédemment. La figure 3 représentent l'évolution de ces deux raies en fonction de la densité d'énergie injectée. 


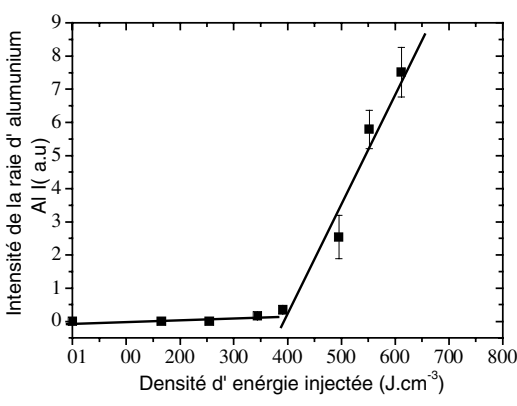

a)

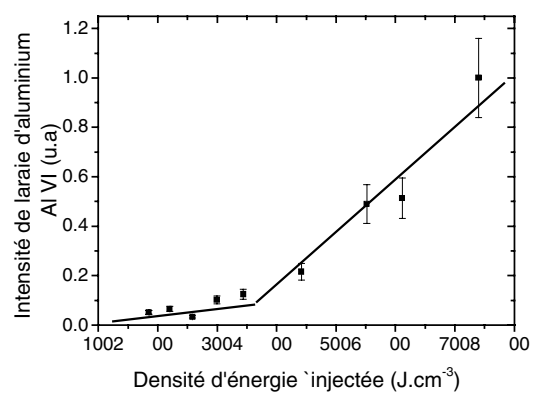

b)

Figure 3. Evolutions des raies d'aluminium Al I et Al VI en fonction de la densité d'énergie transférée au gaz circulant dans le capillaire correspondant respectivement à (a) et (b).

Ces résultats montrent que l'ablation est très dépendante de la densité d'énergie injectée. Quand cette dernière est inférieure à $350 \mathrm{~J} . \mathrm{cm}^{-3}$, l'intensité des raies d'aluminium est très faible. Au delà de cette valeur critique, l'intensité des raies d'aluminium devient très élevée. Il s'agit donc d'un seuil d'ablation situé autour de cette densité d'énergie injectée de $350 \pm 50 \mathrm{~J} . \mathrm{cm}^{-3}$ qui définit deux régimes de fonctionnement de la décharge : un régime ablatif et un régime non ablatif.

\section{4. ÉTUDE DE SEUIL D’ABLATION}

Nous avons étudié l'influence du gaz circulant dans le capillaire sur le seuil d'ablation afin de mieux comprendre ce mécanisme d'ablation. En plus de l'hélium, le xénon $\mathrm{Xe}$ et le propène $\mathrm{C}_{3} \mathrm{H}_{6}$ ont été utilisés. La pression du xénon et du propène dans le capillaire est de 1 mbar. La figure 4 représente les évolutions de l'intensité de la raie d'aluminium Al VI à $30.8 \mathrm{~nm}$ en fonction de la densité d'énergie injectée pour ces trois gaz.

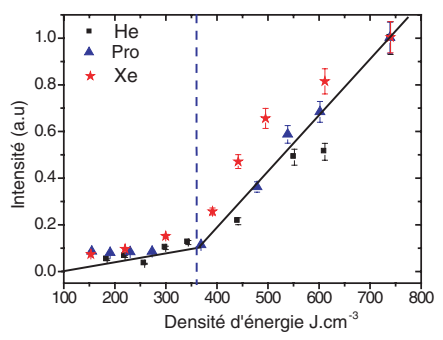

Figure 4. Evolution de l'intensité de la raie d'aluminium Al VI à $30.8 \mathrm{~nm}$ en fonction de la densité d'énergie correspondant à l'hélium, au propène et au xénon.

Nous pouvons constater à partir de cette figure que le seuil d'ablation des parois ne dépend pas de la nature du gaz circulant dans le capillaire. Ceci indique que le mécanisme de chauffage des parois lors de la décharge n'est pas contrôlé principalement par le rayonnement émis par le plasma. En effet, l'émissivité des ces trois gaz est très différente. De plus, la conduction thermique due aux électrons paraît être le principal moteur dans l'interaction entre le plasma et les parois par rapport à la conduction thermique due aux ions. Ceci corrobore les résultats des travaux théoriques rapportés en [3] qui portent sur la simulation d'une décharge dans un capillaire en alumine en flux d'hydrogène. Ils ont permis, entre autre, de déterminer la température des parois du capillaire en fonction du temps et du courant circulant dans le capillaire. Ils ont révélé que la conduction thermique due aux ions joue un rôle secondaire en comparaison de la conduction thermique due aux électrons. 


\section{MODÈLE THERMIQUE}

Le travail présenté en [1] décrit un modèle thermique capable de modéliser les transferts de chaleur entre un plasma créé par décharge et les parois internes d'un capillaire. Il est évident qu'un gradient de température dans un milieu engendre un flux d'énergie thermique dans la direction des températures décroissantes (loi de Fourier). Dans notre cas, le flux de chaleur se propage dans les parois du capillaire. La résolution de l'équation de chaleur, donnée en (1), permet de décrire l'évolution de la température d'un système soumis à des inhomogénéités thermiques.

$$
D \frac{1}{r} \frac{\partial}{\partial r}\left(r \frac{\partial T}{\partial r}\right)=\frac{\partial T}{\partial t}
$$

où $\mathrm{D}$ représente le coefficient de diffusion thermique de l'alumine qui dépend des propriétés thermique du capillaire. Une solution de l'équation (1) a été donnée en [1] en considérant que la densité du flux thermique du plasma créé par la décharge décroît de façon exponentielle en fonction de temps. Dans le cas de notre décharge, cette approximation reste valable car l'évolution de l'énergie dissipée par effet joule dans le plasma peut être enveloppée par une exponentielle décroissante. La densité de flux thermique du plasma $\phi(t)$ peut s'écrire par conséquent sous la forme suivante :

$$
\phi(t)=\phi_{0} e^{-\frac{t}{\tau}}
$$

avec $\phi_{0}$ la densité de flux thermique initial du plasma et $\tau$ la constante de temps qui caractérise le temps de décroissance caractéristique du flux thermique. Nous trouvons que cette constante $\tau$ est de l'ordre de $600 \mathrm{~ns}$. Il faut signaler que ce temps $\tau$ dépend légèrement de la densité d'énergie injectée mais reste tout de même inférieur à $1 \mu \mathrm{s}$. En tenant compte de l'expression du flux thermique du plasma $\phi(\mathrm{t})$, des conditions aux limites et des conditions initiales, la résolution de l'équation (1) peut être parfaitement accomplie. La température des parois du capillaire en fonction du temps peut donc s'écrire sous la forme suivante :

$$
T\left(a_{0}, t\right)=\frac{\phi_{0} \sqrt{3 D \tau}}{3 \kappa} e^{-\frac{t}{\tau}} \sqrt{e^{\frac{t}{\tau}}-1}
$$

où $\kappa$ représente la conductivité thermique de l'alumine et $\mathrm{a}_{0}$ représente le rayon intérieur du capillaire. Nous remarquons qu'elle dépend principalement de la densité du flux thermique initial du plasma et des propriétés thermiques du matériau constituant le capillaire. Le seuil d'ablation des parois est atteint quand le maximum de la température des parois devient égale à la température de fusion d'alumine qui est de $2323 \mathrm{~K}$. Le flux thermique correspondant au seuil $\phi_{\mathrm{s}}$ peut être déterminé en intégrant l'équation (4) et en remplaçant T par la température de fusion du capillaire $\mathrm{T}_{\mathrm{m}}$. On en déduit que $\phi_{\mathrm{s}} \mathrm{s}$ 'écrit sous la forme suivante :

$$
\phi_{s}=2 C_{p} T_{m} \rho \sqrt{\frac{3 D}{\tau}}
$$

où $\rho$ désigne la densité volumique de l'alumine. Nous trouvons que le flux thermique seuil $\phi_{\mathrm{s}}$ est égale à $12 \mathrm{MW} \cdot \mathrm{cm}^{-2}$. Par conséquence, la densité d'énergie injectée correspondant au seuil est de l'ordre de $300 \mathrm{~J} . \mathrm{cm}^{-3}$. Elle très proche de la densité seuil issue des mesures spectroscopiques.

La figure 5 représente l'évolution de la température des parois pour quatre densités d'énergie injectées correspondant à 200,300, 400 et $600 \mathrm{~J} . \mathrm{cm}^{-3}$. La ligne en pointillée sur la figure indique la température de fusion du capillaire $T_{\mathrm{m}}$ qui est égale à $2323 \mathrm{~K}$. Nous vérifions ainsi que la température de fusion des parois est atteinte pour une densité d'énergie de $300 \mathrm{~J}_{\mathrm{cm}}{ }^{-3}$. La température $\mathrm{T}_{\mathrm{m}}$ est atteinte plus rapidement quand l'énergie injectée devient plus élevée. Dans le cas correspondant à $600{\mathrm{~J} . \mathrm{cm}^{-3}}^{-3}$, la température $\mathrm{T}_{\mathrm{m}}$ est atteinte dès $100 \mathrm{~ns}$ après l'établissement de la décharge, ce qui signifie que l'ablation s'initie aussi à ce même instant. Nous avons signalé précédemment que l'évolution de l'intensité de la raie d'aluminium Al VI à $30.8 \mathrm{~nm}$ est également maximale autour de 


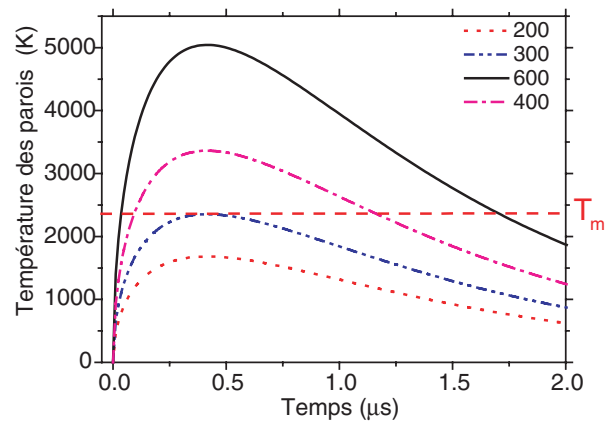

Figure 5. Evolution temporelle de la température des parois en fonction de la densité d'énergie injectée calculé à partir du modèle $[1]\left(\mathrm{D}=10 \mathrm{~m}^{2} \mathrm{~s}^{-1}, \rho=3900 \mathrm{Kg} \cdot \mathrm{cm}^{-3}\right.$ et $\left.\kappa=35 \mathrm{~W} / \mathrm{m} / \mathrm{K}\right)$.

120 ns. Ces résultats du modèle apparaissent en très bon accord avec les résultats issus des mesures spectroscopiques.

\section{CONCLUSION}

Les sources EUV par décharge capillaire représentent un moyen simple et efficace pour produire un plasma chaud, dense et fortement émissif dans la gamme spectrale de l'EUV. Nous nous sommes particulièrement intéressés dans ce travail à étudier l'ablation des parois du capillaire. Le but de cette étude est de contrôler l'ablation du capillaire pour prolonger ainsi la durée de vie de ces sources de rayonnement. Les mesures spectroscopiques dans les domaines spectraux de l'EUV et de l'ultraviolet ont révélé la présence d'un seuil d'ablation des parois situé autour d'une densité d'énergie injectée de $350{\mathrm{~J} . \mathrm{cm}^{-3}}^{-3}$. Ce seuil est indépendant du gaz circulant dans le capillaire. La source peut fonctionner soit en régime ablatif, soit en régime non ablatif.

Enfin l'utilisation d'un modèle thermique simple basé sur la résolution de l'équation de la chaleur a permis de calculer l'évolution temporelle de la température des parois en fonction de la densité d'énergie injectée. A partir de ces résultats, le seuil d'ablation a pu être également calculé. Nous trouvons un très bon accord entre les résultats issus du modèle et ceux obtenus à partir des mesures spectroscopiques.

\section{Remerciements}

Ce travail est soutenu par le Ministère de la Recherche et Technologie (MRT) et également par le Secrétariat d'État à l'Industrie (MINEFI) dans le cadre du programme national français PREUVE et le programme européen EXTUMASK, MEDEA+.

\section{Bibliographie}

[1] Vallée O., Caillard J. and De Izarra C. A model for coaxial ablation by plasma at the heat flux threshold. Surface Review and letters Vol 10 No 1 (2003) 81-85.

[2] Mohanty S.R., Robert E., Dussart R., Viladrosa R., Pouvesle J.-M., Fleurier C., Cachoncinlle Ch.: A novel fast capillary discharge system emitting intense EUV radiation. Possible source for EUV lithography. Microelectronic Engineering 65 (2003) 47-59.

[3] Bobrova N.A., Bulanov S.V., Farina D., Pozzoli R., Razinkova T.L., Sasorov P.V.: Magnetohydrodynamic simulation of capillary plasmas. Plasma Phys. Reps. 24, 1 (1998) 1-8. 